\title{
Front Matter: Volume 8990
}

, "Front Matter: Volume 8990," Proc. SPIE 8990, Silicon Photonics IX, 899001 (4 April 2014); doi: 10.1117/12.2063961

SPIE. Event: SPIE OPTO, 2014, San Francisco, California, United States 


\section{PROCEEDINGS OF SPIE}

\section{Silicon Photonics IX}

Joel Kubby

Graham T. Reed

Editors

3-5 February 2014

San Francisco, California, United States

Sponsored and Published by

SPIE 
The papers included in this volume were part of the technical conference cited on the cover and title page. Papers were selected and subject to review by the editors and conference program committee. Some conference presentations may not be available for publication. The papers published in these proceedings reflect the work and thoughts of the authors and are published herein as submitted. The publisher is not responsible for the validity of the information or for any outcomes resulting from reliance thereon.

Please use the following format to cite material from this book:

Author(s), "Title of Paper," in Silicon Photonics IX, edited by Joel Kubby, Graham T. Reed, Proceedings of SPIE Vol. 8990 (SPIE, Bellingham, WA, 2014) Article CID Number.

ISSN: 0277-786X

ISBN: 9780819499035

Published by

SPIE

P.O. Box 10, Bellingham, Washington 98227-0010 USA

Telephone +1 3606763290 (Pacific Time) · Fax +1 3606471445

SPIE.org

Copyright @ 2014 , Society of Photo-Optical Instrumentation Engineers.

Copying of material in this book for internal or personal use, or for the internal or personal use of specific clients, beyond the fair use provisions granted by the U.S. Copyright Law is authorized by SPIE subject to payment of copying fees. The Transactional Reporting Service base fee for this volume is $\$ 18.00$ per article (or portion thereof), which should be paid directly to the Copyright Clearance Center (CCC), 222 Rosewood Drive, Danvers, MA 01923. Payment may also be made electronically through CCC Online at copyright.com. Other copying for republication, resale, advertising or promotion, or any form of systematic or multiple reproduction of any material in this book is prohibited except with permission in writing from the publisher. The CCC fee code is 0277-786X/14/\$18.00.

Printed in the United States of America.

Publication of record for individual papers is online in the SPIE Digital Library.

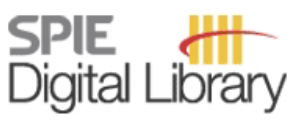

SPIEDigitalLibrary.org

Paper Numbering: Proceedings of SPIE follow an e-First publication model, with papers published first online and then in print and on CD-ROM. Papers are published as they are submitted and meet publication criteria. A unique, consistent, permanent citation identifier (CID) number is assigned to each article at the time of the first publication. Utilization of CIDs allows articles to be fully citable as soon as they are published online, and connects the same identifier to all online, print, and electronic versions of the publication. SPIE uses a six-digit CID article numbering system in which:

- The first four digits correspond to the SPIE volume number.

- The last two digits indicate publication order within the volume using a Base 36 numbering

system employing both numerals and letters. These two-number sets start with 00, 01, 02, 03, 04,

05, 06, 07, 08, 09, 0A, OB ... 0Z, followed by 10-1Z, 20-2Z, etc.

The CID Number appears on each page of the manuscript. The complete citation is used on the first page, and an abbreviated version on subsequent pages. Numbers in the index correspond to the last two digits of the six-digit CID Number. 


\section{Contents}

ix Conference Committee

SESSION 1 WAVEGUIDES I

899002 A compact and low-loss silicon waveguide crossing for O-band optical interconnect [8990-1]

Y. Zhang, Univ. of Delaware (United States); M. Streshinsky, A. Novack, A*STAR Institute of Microelectronics (Singapore) and National Univ. of Singapore (Singapore); Y. Ma, S. Yang, Univ. of Delaware (United States); A. E.-J. Lim, G.-Q. Lo, National Univ. of Singapore (Singapore); T. Baehr-Jones, Univ. of Delaware (United States); M. Hochberg, Univ. of Delaware (United States), A*STAR Institute of Microelectronics (Singapore), and National Univ. of Singapore (Singapore)

899003 Launching of multi-project wafer runs in ePIXfab with micron-scale silicon rib waveguide technology [8990-2]

T. Aalto, M. Cherchi, M. Harjanne, S. Ylinen, M. Kapulainen, T. Vehmas, VTT Technical Research Ctr. of Finland (Finland)

899004 The Euler bend: paving the way for high-density integration on micron-scale semiconductor platforms [8990-3]

M. Cherchi, S. Ylinen, M. Harjanne, M. Kapulainen, T. Vehmas, T. Aalto, VTT Technical Research Ctr. of Finland (Finland)

899005 Low-loss spiral waveguides with ultra-small footprint on a micron scale SOI plafform [8990-4]

M. Cherchi, S. Ylinen, M. Harjanne, M. Kapulainen, T. Vehmas, T. Aalto, VTT Technical Research Ctr. of Finland (Finland)

899006 A new generation of ultra-dense optical I/O for silicon photonics (Invited Paper) [8990-5] M. S. Wlodawski, V. I. Kopp, J. Park, J. Singer, E. E. Hubner, D. Neugroschl, N. Chao, Chiral Photonics, Inc. (United States); A. Z. Genack, Queens College, CUNY (United States)

899007 A high-performance SOI grating coupler with completely vertical emission [8990-6] H.-L. Tseng, C.-W. Tseng, National Tsing Hua Univ. (Taiwan); E. Chen, Forelux Inc. (Taiwan); N. Na, National Tsing Hua Univ. (Taiwan)

\section{SESSION 2 WAVEGUIDES II}

899008 Erasable diffractive grating couplers in silicon on insulator for wafer scale testing [8990-7] R. Topley, G. Martinez-Jimenez, Univ. of Southampton (United Kingdom); L. O'Faolain, Univ. of St. Andrews (United Kingdom); N. Healy, S. Mailis, D. J. Thomson, F. Y. Gardes, A. C. Peacock, D. N. R. Payne, G. Z. Mashanovich, G. T. Reed, Univ. of Southampton (United Kingdom) 
899009 Cost-effective single-etched TM-mode SOI grating couplers for broadband perfectly vertical coupling [8990-8]

G. Dabos, Aristotle Univ. of Thessaloniki (Greece); D. Kalavrouziotis, National Technical Univ. of Athens (Greece); J. Bolten, A. Prinzen, AMO GmbH, AMICA (Germany); N. Pleros, D. Tsiokos, Aristotle Univ. of Thessaloniki (Greece) and Ctr. for Research and Technology Hellas (Greece)

8990 0A Tolerance analysis for efficient MMI devices in silicon photonics [8990-9] C. Vázquez, A. Tapetado, Univ. Carlos III de Madrid (Spain) and Massachusetts Institute of Technology (United States); J. Orcutt, H. C. Meng, R. Ram, Massachusetts Institute of Technology (United States)

\section{SESSION 3 WAVEGUIDES III}

8990 0C Silicon photonic crystals: light emission, modulation and detection (Invited Paper) [8990-11] K. Debnath, A. Shakoor, K. Welna, Univ. of St. Andrews (United Kingdom); F. Gardes, G. T. Reed, Univ. of Southampton (United Kingdom); M. Ferrera, Univ. of St. Andrews (United Kingdom); K. Deasy, D. Lidzey, The Univ. of Sheffield (United Kingdom); R. Lo Savio, Univ. degli Studi di Pavia (Italy); P. Cardile, Univ. of St. Andrews (United Kingdom) and Istituto per la Microelettronica e Microsistemi, CNR and Univ. degli Studi di Catania (Italy); S. Portalupi, D. Gerace, Univ. degli Studi di Pavia (Italy); S. Boninelli, G. Franzó, F. Priolo, Istituto per la Microelettronica e Microsistemi, CNR and Univ. degli Studi di Catania (Italy); A. P. Knights, McMaster Univ. (Canada); M. Galli, Univ. degli Studi di Pavia (Italy); T. F. Krauss, Univ. of St. Andrews (United Kingdom) and Univ. of York (United Kingdom); L. O'Faolain, Univ. of St. Andrews (United Kingdom)

$8990 \mathrm{OE} \quad$ The evolution of angled MMI structure on the SOI platform [8990-13] Y. Hu, D. J. Thomson, F. Y. Gardes, G. Z. Mashanovich, G. T. Reed, Univ. of Southampton (United Kingdom)

8990 OF Fabrication-tolerant optical filters for dense integration on a micron-scale SOI platform [8990-14]

M. Cherchi, S. Ylinen, M. Harjanne, M. Kapulainen, T. Vehmas, T. Aalto, VTT Technical Research Ctr. of Finland (Finland); G. T. Kanellos, Ctr. for Research and Technology Hellas (Greece); D. Fitsios, Ctr. for Research and Technology Hellas (Greece) and Aristotle Univ. of Thessaloniki (Greece); N. Pleros, Ctr. for Research and Technology Hellas (Greece)

8990 0G Apodized grating silicon waveguides for tunable optical delay lines [8990-15] S. Khan, S. Fathpour, CREOL, The College of Optics and Photonics, Univ. of Central Florida (United States) and Univ. of Central Florida (United States)

$8990 \mathrm{OH} \quad$ Silicon nanomembrane-based compact true-time-delay module on unconventional substrates [8990-16]

H. Subbaraman, X. Xu, Omega Optics, Inc. (United States); R. T. Chen, The Univ. of Texas at Austin (United States) 
8990 OK Characteristics of avalanche electroluminescent nanoscale Si light sources in SOI technology [8990-19]

C. Janse van Rensburg, INSiAVA (Pty) Ltd. (South Africa); M. du Plessis, P. J. Venter, Univ. of Pretoria (South Africa)

8990 OL High-intensity 100-nW 5-GHz silicon avalanche LED utilizing carrier energy and momentum engineering [8990-20]

L. W. Snyman, Tshwane Univ. of Technology (South Africa); J.-L. Polleux, Univ. Paris-Est Marne-la-Vallée (France); K. A. Ogudo, Tshwane Univ. of Technology (South Africa);

C. Viana, S. Wahl, Univ. Paris-Est Marne-la-Vallée (France)

8990 ON Dual-facet coupling of SOA array on 4- $\mu$ m silicon-on-insulator implementing a hybrid integrated SOA-MZI wavelength converter [8990-22]

T. Alexoudi, D. Fitsios, Ctr. for Research and Technology Hellas (Greece) and Aristotle Univ. of Thessaloniki (Greece); G. T. Kanellos, Ctr. for Research and Technology Hellas (Greece); N. Pleros, Ctr. for Research and Technology Hellas (Greece) and Aristotle Univ. of Thessaloniki (Greece); T. Tekin, Technische Univ. Berlin (Germany); M. Cherchi, S. Ylinen, M. Harjanne, M. Kapulainen, T. Aalto, VTT Technical Research Ctr. of Finland (Finland)

$899000 \quad$ Mid-infrared nonlinear silicon photonics (Invited Paper) [8990-23]

X. Liu, Nanjing Univ. (China); B. Kuyken, Univ. Gent (Belgium); W. M. J. Green, IBM Thomas

J. Watson Research Ctr. (United States); R. M. Osgood Jr., Columbia Univ. (United States);

R. Baets, G. Roelkens, IBM Thomas J. Watson Research Ctr. (United States)

SESSION $5 \quad$ LAB-ON-A-CHIP I

8990 OR Ultrasensitive optofluidic-nanoplasmonic BioNEMS for life sciences and point-of-care diagnostics (Invited Paper) [8990-25]

A. A. Yanik, Univ. of California, Santa Cruz (United States)

8990 OS Methods to array photonic crystal microcavities for high throughput high sensitivity biosensing on a silicon-chip based platform (Invited Paper) [8990-26]

S. Chakravarty, H. Subbaraman, Omega Optics, Inc. (United States); Y. Zou, W.-C. Lai, The Univ. of Texas at Austin (United States); R. T. Chen, Omega Optics, Inc. (United States) and The Univ. of Texas at Austin (United States)

8990 OT Silicon photomultipliers applications to biosensors [8990-27]

M. F. Santangelo, R. Pagano, S. Lombardo, Istituto per la Microelettronica e Microsistemi, CNR (Italy); E. L. Sciuto, Univ. degli Studi di Catania (Italy); D. Sanfilippo, G. Fallica,

STMicroelectronics (Italy); F. Sinatra, Univ. degli Studi di Catania (Italy); S. Libertino, Istituto per la Microelettronica e Microsistemi, CNR (Italy)

8990 OU High-performance conformal sensors employing single-crystal silicon nanomembranes [8990-28]

X. Xu, H. Subbaraman, S. Chakravarty, Omega Optics, Inc. (United States); R. T. Chen, The Univ. of Texas at Austin (United States) 
$89900 \mathrm{~V}$ Integrated silicon microring resonator devices for point-of-care diagnostic applications (Invited Paper) [8990-29]

M. K. Park, Q. Liu, K. W. Kim, Y. Shin, J. S. Kee, J. Song, G.-Q. Lo, D.-L. Kwong, A*STAR Institute of Microelectronics (Singapore)

8990 OW Sensing platform based on micro-ring resonator and on-chip reference sensors in SOI [8990-30]

S. M. C. Abdulla, B. M. de Boer, J. M. Pozo, J. H. van den Berg, A. Abutan, R. A. J. Hagen,

D. M. R. LoCascio, P. J. Harmsma, TNO (Netherlands)

8990 0X Integrated strip and slot waveguides in silicon-on-sapphire for mid infrared VOC detection in water [8990-31]

Y. Zou, The Univ. of Texas at Austin (United States); H. Subbaraman, S. Chakravarty, X. XU, A. Hosseini, Omega Optics, Inc. (United States); W.-C. Lai, The Univ. of Texas at Austin (United States); R. T. Chen, The Univ. of Texas at Austin (United States) and Omega Optics, Inc. (United States)

$8990 \mathrm{OZ}$ Comparative sensitivity analysis of integrated optical waveguides for near-infrared volatile organic compounds with 1 ppb detection [8990-33]

W.-C. Lai, Y. Zou, The Univ. of Texas at Austin (United States); S. Chakravarty, Omega Optics, Inc. (United States); L. Zhu, The Univ. of Texas at Austin (United States); R. T. Chen, The Univ. of Texas at Austin (United States) and Omega Optics, Inc. (United States)

\section{SESSION 7 MODULATORS}

899010 High-speed silicon-based integrated optical modulators for optical-fiber telecommunications (Invited Paper) [8990-34]

K. Ogawa, Fujikura Ltd. (Japan)

899011 High-contrast and accurate high-speed simulation of silicon-based modulators [8990-35] C. E. Png, M. J. Sun, S. T. Lim, A*STAR Institute of High Performance Computing (Singapore); D. Thomson, Univ. of Southampton (United Kingdom)

$899012 \quad$ 40-Gbit/s silicon modulators fabricated on $\mathbf{2 0 0}-\mathrm{mm}$ and $\mathbf{3 0 0}-\mathrm{mm}$ SOI wafers [8990-36] D. Marris-Morini, Institut d'Électronique Fondamentale, CNRS, Univ. Paris-Sud (France); C. Baudot, STMicroelectronics (France); J.-M. Fédéli, CEA-LETI (France); G. Rasigade, Institut d'Électronique Fondamentale, CNRS, Univ. Paris-Sud (France); N. Vuillet, STMicroelectronics (France); A. Souhaité, STMicroelectronics (France) and CEA-LETI (France); M. Ziebell, P. Rivalin, Institut d'Électronique Fondamentale, CNRS, Univ. Paris-Sud (France); S. Olivier, CEA-LETI (France); P. Crozat, D. Bouville, Institut d'Électronique Fondamentale, CNRS, Univ. Paris-Sud (France); S. Menezo, STMicroelectronics (France); F. Boeuf, Institut d'Électronique Fondamentale, CNRS, Univ. Paris-Sud (France); L. Vivien, CEA-LETI (France) 
899013 Silicon high speed modulator for advanced modulation: device structures and exemplary modulator performance (Invited Paper) [8990-37]

B. Milivojevic, S. Wiese, J. Whiteaway, C. Raabe, Cisco Optical GmbH (Germany);

A. Shastri, Stanford Univ. (United States) and Cisco Systems Inc. (United States); M. Webster, P. Metz, S. Sunder, B. Chattin, S. P. Anderson, B. Dama, K. Shastri, Cisco Systems, Inc. (United States)

\section{SESSION 8 DETECTORS}

899014 Rapid-melt-growth-based GeSi waveguide photodetectors and avalanche photodetectors (Invited Paper) [8990-38]

N. Na, C.-K. Tseng, National Tsing Hua Univ. (Taiwan); Y. Kang, Intel Corp. (United States); M.-C. M. Lee, National Tsing Hua Univ. (Taiwan)

899015 Low-cost radioactivity monitoring with scintillating fibers and silicon photomultipliers [8990-39]

P. Finocchiaro, L. Cosentino, A. Pappalardo, C. Scirè, S. Scirè, G. Vecchio, Istituto Nazionale di Fisica Nucleare (Italy)

899016 Design and development of a fNIRS system prototype based on SiPM detectors [8990-40] D. Sanfilippo, G. Valvo, M. Mazzillo, A. Piana, B. Carbone, L. Renna, P. G. Fallica, STMicroelectronics (Italy); D. Agrò, G. Morsellino, M. Pinto, R. Canicattì, N. Galioto, A. Tomasino, G. Adamo, S. Stivala, A. Parisi, L. Curcio, C. Giaconia, A. C. Busacca, Univ. degli Studi di Palermo (Italy); R. Pagano, S. Libertino, S. Lombardo, Istituto per la Microelettronica e Microsistemi, CNR (Italy)

899017 Responsivity measurements of $\mathbf{4 H}-\mathrm{SiC}$ Schottky photodiodes for UV light monitoring [8990-41]

G. Adamo, D. Agrò, S. Stivala, A. Parisi, L. Curcio, A. Andò, A. Tomasino, C. Giaconia, A. C. Busacca, Univ. degli Studi di Palermo (Italy); M. C. Mazzillo, D. Sanfilippo, P. G. Fallica, STMicroelectronics (Italy)

899018 Potentialities of silicon photomultiplier [8990-42]

R. Pagano, S. Libertino, D. Corso, Istituto per la Microelettronica e Microsistemi, CNR (Italy); G. Valvo, D. Sanfilippo, P. G. Fallica, STMicroelectronics (Italy); S. Lombardo, Istituto per la Microelettronica e Microsistemi, CNR (Italy)

899019 SNR measurements of silicon photomultipliers in the continuous wave regime [8990-43] G. Adamo, D. Agrò, S. Stivala, A. Parisi, C. Giaconia, A. C. Busacca, Univ. degli Studi di Palermo (Italy); G. Fallica, STMicroelectronics (Italy) 


\section{SESSION 9 RESONATORS}

8990 1C Strain engineering in germanium microdisks [8990-46]

A. Ghrib, M. El Kurdi, Institut d'Électronique Fondamentale, CNRS, Univ. Paris-Sud (France); M. Prost, Institut d'Électronique Fondamentale, CNRS, Univ. Paris-Sud (France) and STMicroelectronics (France); M. de Kersauson, Institut d'Électronique Fondamentale, CNRS, Univ. Paris-Sud (France); L. Largeau, O. Mauguin, G. Beaudoin, Lab. de Photonique et de Nanostructures, CNRS (France); S. Sauvage, X. Checoury, Institut d'Électronique Fondamentale, CNRS, Univ. Paris-Sud (France); G. Ndong, M. Chaigneau, R. Ossikovski, Lab. de Physique des Interfaces et des Couches Minces, CNRS, Ecole Polytechnique (France); S. David, Lab. des Technologies de la Microélectronique, CNRS, Univ. Joseph Fourier (France); I. Sagnes, Lab. de Photonique et de Nanostructures, CNRS (France); P. Boucaud, Institut d'Électronique Fondamentale, CNRS, Univ. Paris-Sud (France)

8990 1D Low-loss and flatband silicon-nanowire-based 5th-order coupled resonator optical waveguides (CROW) fabricated by ArF-immersion lithography process on a $300-\mathrm{mm}$ SOI wafer [8990-47]

S.-H. Jeong, D. Shimura, T. Simoyama, Photonics Electronics Technology Research Association (Japan); M. Seki, N. Yokoyama, M. Ohtsuka, K. Koshino, National Institute of Advanced Industrial Science and Technology (Japan); T. Horikawa, Photonics Electronics Technology Research Association (Japan) and National Institute of Advanced Industrial Science and Technology (Japan); Y. Tanaka, K. Morito, Photonics Electronics Technology Research Association (Japan)

8990 IE Chirped photonic crystal mode converters for broad-band coupling with highly dispersive photonic crystal microring resonators [8990-48]

S. M. Lo, Vanderbilt Univ. (United States); J. Y. Lee, Univ. of Rochester (United States);

S. M. Weiss, Vanderbilt Univ. (United States); P. M. Fauchet, Vanderbilt Univ. (United States) and Univ. of Rochester (United States)

8990 IF Self-coupled optical waveguide (SCOW) resonators for optical signal processing (Invited Paper) [8990-49]

L. Zhou, S. Li, J. Xie, Q. Wu, J. Chen, Shanghai Jiao Tong Univ. (China)

\section{POSTER SESSION}

8990 IG Hybrid integrated InGaAsP-Si laser using selective area metal bonding method for optical interconnection [8990-50]

H.-Y. YU, L.-J. Yuan, Institute of Semiconductors (China); L. Tao, Peking Univ. (China); B.-J. Wang, Institute of Semiconductors (China); W.-X. Chen, Peking Univ. (China); S. Liang, Institute of Semiconductors (China); Y.-P. Li, G.-Z. Ran, Peking Univ. (China); J.-Q. Pan, W. Wang, Institute of Semiconductors (China)

$8990 \mathrm{lH} \quad$ Silicon Mach-Zehnder interferometer racetrack microring for sensing [8990-51] Y. Xiong, W. N. Ye, Carleton Univ. (Canada)

Author Index 


\section{Conference Committee}

Symposium Chairs

David L. Andrews, University of East Anglia Norwich (United Kingdom)

Alexei L. Glebov, OptiGrate Corporation (United States)

Symposium Co-chairs

Jean Emmanuel Broquin, IMEP-LAHC (France)

Shibin Jiang, AdValue Photonics, Inc. (United States)

Program Track Chair

Yakov Sidorin, Quarles \& Brady LLP (United States)

Conference Chairs

Joel Kubby, University of California, Santa Cruz (United States)

Graham T. Reed, University of Southampton (United Kingdom)

Conference Program Committee

Laurence W. Cahill, La Trobe University (Australia)

Philippe M. Fauchet, Vanderbilt University (United States)

L. Cary Gunn, Genalyte, Inc. (United States)

Siegfried Janz, National Research Council Canada (Canada)

Andrew P. Knights, McMaster University (Canada)

Laura Maria Lechuga, Catalan Institute of Nanoscience and Nanotechnology (Spain)

Sebania Libertino, Istituto per la Microelettronica e Microsistemi (Italy)

Goran Z. Mashanovich, University of Southampton (United Kingdom)

Ching Eng J. Png, A*STAR Institute of High Performance Computing (Singapore)

Andrew W. Poon, Hong Kong University of Science and Technology (Hong Kong, China)

Haisheng Rong, Intel Corporation (United States)

Holger Schmidt, University of California, Santa Cruz (United States)

Dan-Xia Xu, National Research Council Canada (Canada)

Zhiping Zhou, Peking University (China) 
Session Chairs

Waveguides I

Graham T. Reed, University of Southampton (United Kingdom)

Waveguides II

Graham T. Reed, University of Southampton (United Kingdom)

Waveguides III

Edgar Huante-Ceron, McMaster University (Canada)

Lab-on-a-Chip I

Hong Cai, University of California, Santa Cruz (United States)

Lab-on-a-Chip II

L. Cary Gunn, Genalyte, Inc. (United States)

Modulators

Haisheng Rong, Intel Corporation (United States)

Detectors

Ching Eng J. Png, A*STAR Institute of High Performance Computing (Singapore) 